\title{
Correction to: Solution methods for linear discrete ill-posed problems for color image restoration
}

\author{
A. H. Bentbib ${ }^{1}$ - M. El Guide ${ }^{1,2}$ - K. Jbilou ${ }^{3}$. \\ E. Onunwor ${ }^{4,5}$. L. Reichel ${ }^{4}$
}

Published online: 4 May 2018

(C) Springer Science+Business Media B.V., part of Springer Nature 2018

\section{Correction to: Bit Numer Math https://doi.org/10.1007/s10543-018-0706-0}

The original version of this article unfortunately contained a mistake. The presentation of Algorithm 4 was incorrect in this article. The corrected Algorithm 4 is given below.

The original article can be found online at https://doi.org/10.1007/s10543-018-0706-0.

$凶 \quad$ L. Reichel

reichel@math.kent.edu

A. H. Bentbib

a.bentbib@uca.ac.ma

M. El Guide

mohamed.elguide@um6p.ma; mohamed.elguide@edu.uca.ac.ma

K. Jbilou

jbilou@univ-littoral.fr

E. Onunwor

eonunwor@starkstate.edu

1 Laboratoire de Mathématiques Appliquées et Informatique, Faculté des Sciences et Techniques-Gueliz, Marrakesh, Morocco

2 Université Mohammed VI Polytechnique, FAB-LAB, UM6P, Bengeurir, Morocco

3 Université du Littoral Côte d'Opale, L.M.P.A, ULCO, 50 rue F. Buisson, BP699, 62228 Calais-Cedex, France

4 Department of Mathematical Sciences, Kent State University, Kent, OH 44242, USA

5 Department of Mathematics, Stark State College, 6200 Frank Ave. NW, North Canton, OH 44720, USA 


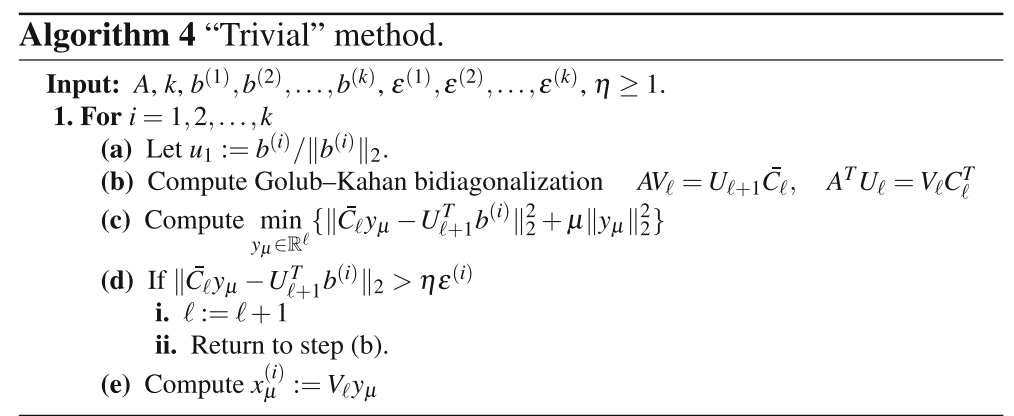

The original article has been corrected.

The publisher sincerely apologizes for this mistake. 